\title{
Human Abnormal Behavior Detection Based on Region Optical Flow Energy
}

\author{
Jian Liu ${ }^{a *}$, Lin Qiu ${ }^{b}$ and Enyang Gao ${ }^{c}$ \\ College of Information and Engineering, Shenyang Jianzhu University,Shenyang 110168, China \\ ajeanliu10@153.com, ${ }^{\mathrm{b}} \mathrm{QiuL}$ _CN@163.com, enyanggao@hotmail.com
}

\begin{abstract}
Keywords: Anomaly detection; Foreground extraction; Vibe algorithm; Optical flow calculation; Energy histogram
\end{abstract}

\begin{abstract}
In the light of the hysteresis of video surveillance call video in public places, as well as the current video surveillance function of a single, in order to improve the real-time performance of monitoring, a detection method of human abnormal behavior based on optical flow method is proposed. The foreground regions of the video sequence is obtained by Vibe algorithm and update the background model adaptively; Regional markers for the future by the nearest neighbor method, accurately extract moving regions; Optical flow of moving region is calculated by Lucas-Kanada method; Weighted energy histogram is used to describe the abnormal changes of human behavior. The video sequences of different scenarios are simulated; the experimental results verify the effectiveness of the proposed method.
\end{abstract}

\section{Introduction}

With the rapid development of computer technology, especially the widespread of computer vision research, video surveillance has spread to every corner of life. But in the present, video surveillance is still very greatly depends on the strength of the artificial recognition and judgment, cannot achieve the real-time monitoring and analysis of one hundred percent discrimination, when happen some problems and it can only find slowly in the massive video database, spending a lot of manpower and resources, reducing the public safety and efficiency greatly. Therefore, the real-time detection and video analysis has important significance for intelligent monitoring, the computer "active" to identify and determine the video's abnormal behavior is an important research content.

Human behavior recognition is the hotspot and difficulty in the research of computer vision, most of the research is still focused on identifying the routine [1-2], but the detection and recognition of abnormal behavior is relatively less. Compare to the normal behavior, chasing, falling, fighting and other typical abnormal behavior is often contain unpredictable, bursty, no periodicity and some other characteristics, so the method of detection and identification of abnormal behavior requirements with strong robustness. In currently, the study of deviance analysis focuses on two aspects: 1) The analysis method based on motion track[3-6], modeling and analysis based on moving target trajectory, so that to determine and predict abnormal behavior. But to obtain relatively accurate human motion trajectory based on accurate and stable human tracking. But due to the influence of the objective environment, this method has obvious limitations; 2) The analysis method based on the characteristics of the human body[7-10], the point of this method is to extract the key parts of the human body, the outline and the direction of motion to describe human motion behavior. But in the process of extraction, this method is easily affected by noise which it is difficult to extract.

This essay is aiming at the limitation of abnormal behavior of current research methods with low accuracy, put forward a kind of abnormal behavior detection method based on regional energy flow. Extract the foreground areas from the video sequence by ViBe algorithm[11-14], then using the nearest neighbor method to mark the foreground regions; Calculate the optical flow motion region, and on the regional energy flow weighted, through the weighted proportion of energy flow to determine the behavior whether it is abnormal. And through the video sequence that in different scenarios to test to verify the validity of this method. 


\section{Vibe Algorithm to Extract the Foreground Object}

$\mathrm{ViBe}$ algorithm is a pixel level video background modeling algorithm. The idea of ViBe is for each pixel stores a sample set, the samples of sampling value is the pixel value of the pixel in the past and its neighboring pixel values, then each new pixel value and sample set were compared to determine whether the point belongs to the background. In ViBe algorithm, background model for each point stored in a sample set, and then each new pixel value and sample set were compared to determine whether the point belongs to the background.

The initialization process of ViBe algorithm only need a frame image, and it is a process of filling the pixel sample set. For a pixel, random choice of its neighbor pixel values as its model sample value.M0(x) $=\{\mathrm{v} 0(\mathrm{y} \mid \mathrm{y} \in \mathrm{NG}(\mathrm{x}))\}, \mathrm{t}=0$ denote its initial time, and $\mathrm{NG}(\mathrm{x})$ is the neighbor point.

The background model is updated so that the background model can adapt to the changing background, selected sample value is updated randomly. In the sample space $\mathrm{N}$, the rate of random probability update makes sample values at time $t$ is not updated in random is $(\mathrm{N}-1) / \mathrm{N}$. If time is continuous, after the time of $\mathrm{dt}$, the rate of sample value remain is:

$$
P(t+d t)=\left(\frac{N-1}{N}\right)^{(t+d t)-t} \text {. }
$$

or

$$
P(t+d t)=e^{-\ln \left(\frac{N}{N-1}\right) d t}
$$

This indicates that a sample value is replaced with time independent $\mathrm{T}$ in the model, and the random strategy is appropriate.

\section{Typical Abnormal Behavior's Optical Flow Energy Description}

The Calculation of Region's Optical Flow. In this paper, Lcaus-Kanada algorithm[16] is used to compute optical flow. Let $\mathrm{m} \times \mathrm{m}$ feature window's optical flow is $(\mathrm{u}, \mathrm{v})$, and in this paper $\mathrm{m}=8$. In the optical flow constraint conditions: $\mathrm{Ixu}+\mathrm{Iyv}+\mathrm{It}=0$, it can get the formula (3)

$$
\left[\begin{array}{cc}
I_{x 1} & I_{y 1} \\
I_{x 2} & I_{y 2} \\
\cdots & \cdots \\
I_{x n} & I_{y n}
\end{array}\right]\left[\begin{array}{l}
u \\
v
\end{array}\right]=-\left[\begin{array}{c}
I_{t 1} \\
I_{t 2} \\
\cdots \\
I_{t n}
\end{array}\right] .
$$

In the formula $3, \mathrm{n}$ is the number of pixels in the feature window $(\mathrm{n}=\mathrm{m} 2)$, Ix and Iy separately representing the image gradient, It is image's time gradient. The equation (3) can be launched:

$$
\left[\begin{array}{l}
u \\
v
\end{array}\right]=\left[\begin{array}{cc}
\sum I_{x_{i}}^{2} & \sum I_{x_{i}} I_{y_{i}} \\
\sum I_{x_{i}} I_{y_{i}} & \sum I_{y_{i}}^{2}
\end{array}\right]^{-1}\left[\begin{array}{l}
-\sum I_{x_{i}} I_{t i} \\
-\sum I_{y_{i}} I_{t i}
\end{array}\right] .
$$

In the equation (4), complexity of regional optical flow computation is $O(\eta N / m 2), \eta$ is prospects for the proportion of the whole image, $\mathrm{N}$ represents the total number of image pixels, $\mathrm{m} 2$ represents the size of characteristics window. According to the formula (5) of energy flow.

$$
E_{k}(n)=\sum_{i=1}^{W} \sum_{j=1}^{H} w_{i, j}(n) v_{i, j}^{2}(n)
$$

Extracting the moving target's optical flow energy of the $\mathrm{N}$ frame image in the video. $\mathrm{E}_{\mathrm{k}}(\mathrm{n})$ 
represent the K sports regional energy flow, $v_{\mathrm{i}, \mathrm{j}}(\mathrm{n})$ represent the $\mathrm{n}$ frame image pix (i, j)'s speed, $w_{\mathrm{i}, \mathrm{j}}(\mathrm{n})$ represent optical energy weight, $\mathrm{W}$ represent movement region width, $\mathrm{H}$ represent movement region height. The speed of each pixel in movement region as shown in equation (6)

$$
v_{i, j}(n)=\sqrt{\left(\Delta X_{i, j}\right)\left(\Delta Y_{i, j}\right)} .
$$

and $\Delta \mathrm{Xi}, \mathrm{j}, \Delta \mathrm{Yi}, \mathrm{j}$ represent pix (i, j)'s offset in horizontal and vertical direction.

Two Definitions. Definition 1 The weights of energy flow:

$$
w_{i, j}(n)=\left(\frac{\Delta \text { Angel }}{\pi} \times \alpha\right)^{2}+\left(\frac{\Delta \text { AngelMax }}{\pi} \times \alpha\right)^{2} .
$$

In formula (7)

$$
\Delta \text { Angel }=\mid \angle \text { AngelCurr }-\angle \text { AngelAvg } \mid .
$$

$$
\triangle \text { AngelMax }=\mid \angle \text { AngelCurr }-\angle \text { AngelMax } \mid .
$$

And $\angle$ AngelCurr represent the angle between the current pixel and the horizontal direction; $\angle$ AngelMax represent the angle of the maximum speed pixel point in movement region; $\angle$ AngelAvg represent the average value of the moving object and the horizontal angle:

$$
\angle \text { AngelAvg }=\frac{\sum \text { Angel }_{i, j}}{\operatorname{Sum}(P)} .
$$

In formula (10), Sum (P) represent the sum of all pixel in motion region; $\alpha$ represent the weight that can adjust the flow of energy. In order to distinguish normal behavior and abnormal behavior more effectively, the weights of energy flow should be selected as large. In this paper $\alpha=12$.

Definition 2 Unit of energy flow:

$$
\overline{E_{k}(n)}=\frac{E_{k}(n)}{\operatorname{Sum}_{k}(p)}
$$

Due to unit of energy flow is a reflection of the intensity of the energy flow of target region, so never considering the optical flow, indoor and outdoor environment change effects on moving objects, and never considering the camera movement.

\section{Results and Discussion}

Vibe Foreground Extraction. At present, the most commonly used method of regional foreground extraction are the frame difference method and background subtraction method. The key of background subtraction method is establish a robust background model (background image). There are some commonly used method to establish background model: Mean value method; Median method; Moving average filter; Gauss; Mixed Gauss model[15]; Codebook and so on. Frame difference method does not require modeling, because its background model is the image of last frame, so that compared with other algorithms the speed relatively fast. But frame difference method to light that transform slowly is not very sensitive, and there are some limitations. 


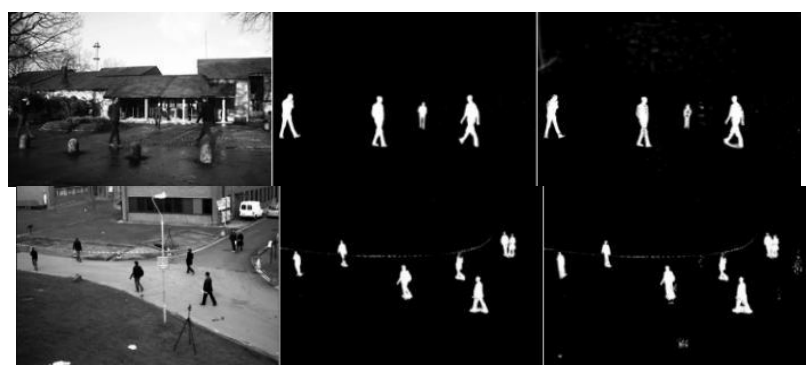

(a) Video frame (b) ViBe algorithm (c) Frame difference

\section{Figure 1. Contrast Foreground extraction}

ViBe algorithm could not only reduce the process of background model, but also could deal a sudden change in the background. When test the background has obviously changes, only need to abandon the original model, reuse the first frame image which has changed to establish the background model. Compared with the mixed Gauss model, frame difference method, ViBe algorithm could update the background more quickly, and need no parameter. The effect is better than the other foreground extraction algorithms. Select the same video frame, and use the ViBe algorithm and the frame difference method to get the foreground image. Comparison of extraction effect for ViBe algorithm and frame difference method in Fig. 1.

Motion Region Marking. After getting the foreground completely, using the nearest neighbor method to mark the region. If the I frame hypothesis video frame with $\mathrm{P}$ motion region, and each region of the center is $R_{\mathrm{p}}^{\mathrm{i}}=\left[x_{c p}^{i}, y_{c p}^{i}\right]$, and $\mathrm{P}=1,2 \ldots \mathrm{m}$. The $\mathrm{I}-1$ frame has $\mathrm{Q}$ motion region. According to the principle of the nearest neighbor can be obtained, we can get equation (12)

$$
D_{\min }=\operatorname{argmin}\left\|R_{\mathrm{p}}^{\mathrm{i}}-R_{\mathrm{q}}^{\mathrm{i}-1}\right\| \text {. }
$$

In equation (12), $\mathrm{p}=1,2, \ldots, \mathrm{P}, \mathrm{q}=1,2, \ldots, \mathrm{Q}$.

Use the first $\mathrm{p}$ region of the I frame and the upper one, Mahalaobis the shortest distance associated, to achieve regional markers. The result are shown in Fig. 2,(b)

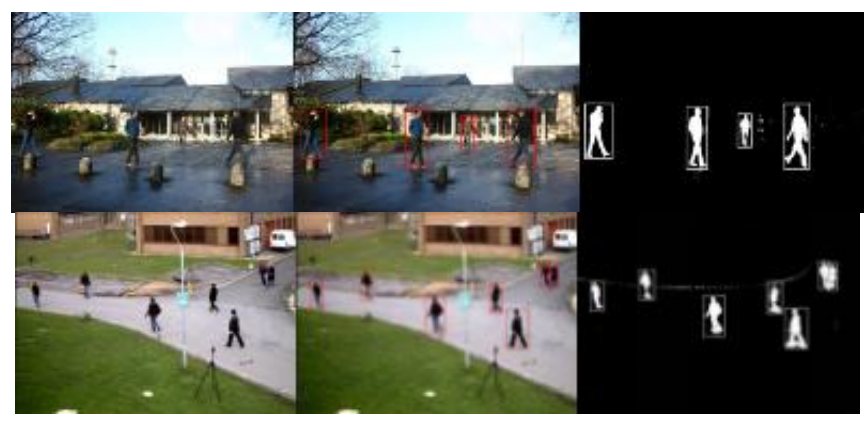

(a) video frame (b) Region marking (c) foreground region marking Figure 2. Motion region marking

Regional Flow Extraction. Calculating optical flow of energy through the region marking the motion region. As shown in Fig. 3, is different behaviors of the regional flow extraction effect 


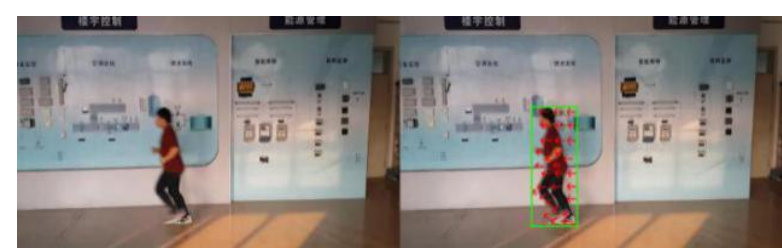

(a) Running

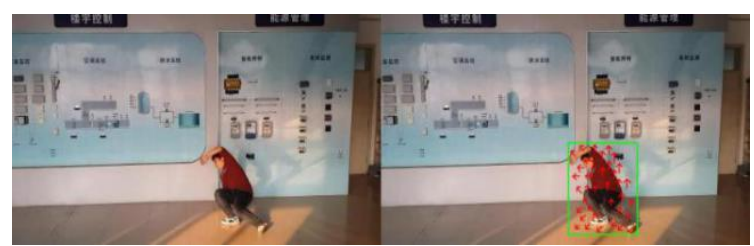

(c) Falling

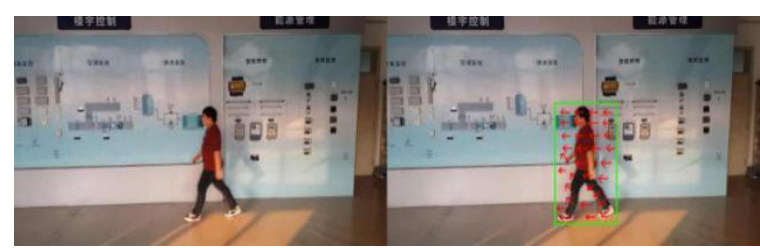

(b) Walking

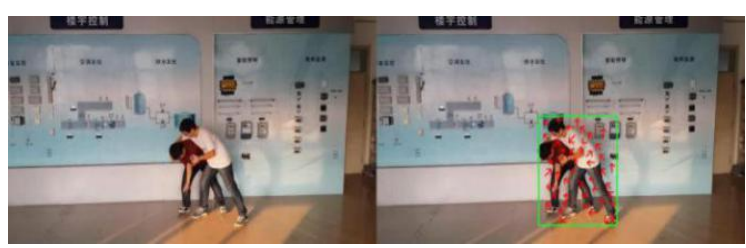

(d) Fighting

Figure. 3 Effect of optical flow in different regions

The Typical Behavior of the Weighted Energy Flow. Compared to the normal behavior, abnormal behavior usually has a large range of movement, the movement direction of clutter and fast moving speed characteristics, the energy movement in one direction higher than the surrounding; And the normal behavior of general motion, roughly the same direction, the movement of energy in one direction is relatively low. So using optical flow reflect the behavior of moving target. Finding the key choice contains different behavior video sequences in indoor, such as falling,walking,chasing and fighting. The selected video frames in the scene storage noise, optical flow changes and other factors. As shown in Fig. 4.

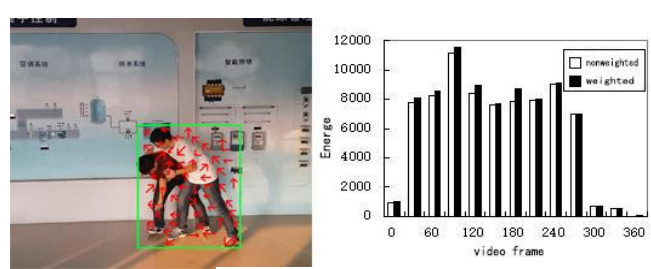

(a) Fighting

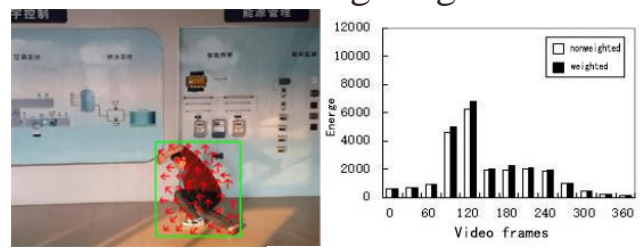

(c) Falling

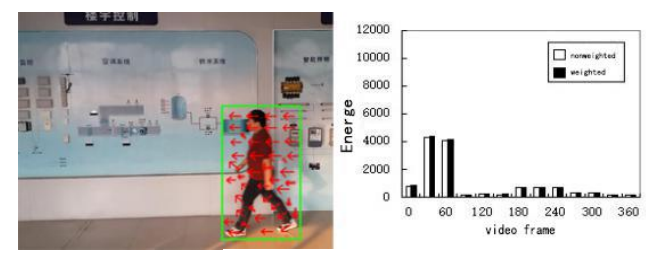

(b) Walking

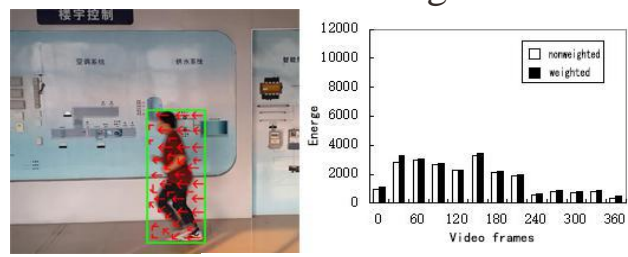

(d) Running

Figure 4. Typical behavior region marking and histogram

As mentioned above, abnormal behavior usually has a large range of movement, the movement direction of clutter and fast moving speed characteristics. So, in a relatively flat area of energy, abnormal behavior of the energy amplitude is much higher than ambient energy amplitude. As shown in Fig. 4, The more violent behavior, the stronger the energy flow, also weighted energy flow is more acutely reflect human behavior's energy changes than non weighted energy flow, and distinguish for wrestling, fighting and other violent behavior more correctly; But non weighted unit of energy flow for the energy change rate is relatively small, could produce false positives or false negatives. The accuracy and speed of video processing to detect abnormal behavior as shown in Table 1. 
Table 1 Video frame behavior detection processing results

\begin{tabular}{ccccc}
\hline Video type & $\begin{array}{c}\text { Video } \\
\text { Number }\end{array}$ & $\begin{array}{c}\text { Alarming } \\
\text { number }\end{array}$ & $\begin{array}{c}\text { Detection } \\
\text { rate[\%] }\end{array}$ & $\begin{array}{c}\text { Average processing } \\
\text { time[ms] }\end{array}$ \\
\hline Outdoor walking & 27 & 1 & 96.30 & 27.3921 \\
Indoor falling & 23 & 18 & 78.26 & 27.7403 \\
Talking & 16 & 2 & 87.5 & 27.5028 \\
Outdoor fighting & 34 & 32 & 94.12 & 26.8433 \\
Indoor fighting & 30 & 30 & 100 & 26.7952 \\
\hline
\end{tabular}

At the same time, this essay compare to document [17] which uses the same optical flow behavior anomaly detection. Fig. 5, (a) (b) (c) is show for indoor environment chasing, fighting, falling flow graph entropy. Shown from the figure, several typical behavior of the flow graph entropy are closed, so that the fighting or chasing occurred it is difficult to distinguish, and easily to produce false positives or false negatives. The results are shown in Table 2.
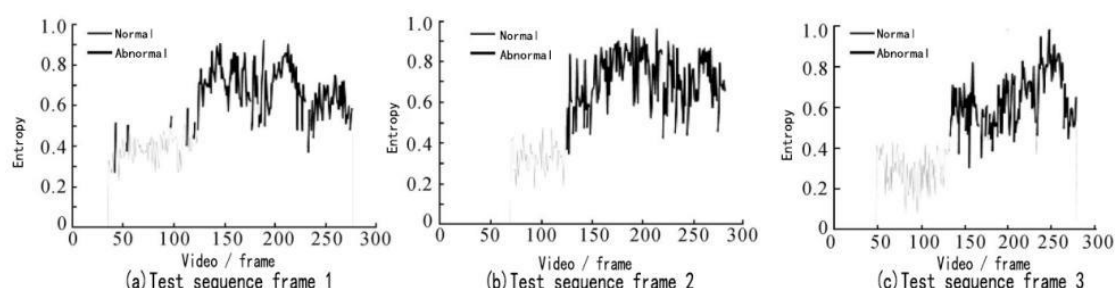

Figure 5. The typical behavior of flow graph entropy in document [17]

Table 2 Comparison result of this algorithm and algorithm for document [17]

\begin{tabular}{|c|c|c|c|c|c|}
\hline \multirow[b]{2}{*}{$\begin{array}{c}\text { Video } \\
\text { type }\end{array}$} & \multirow[b]{2}{*}{$\begin{array}{c}\text { Video } \\
\text { Number }\end{array}$} & \multicolumn{2}{|c|}{ Algorithm in this paper } & \multicolumn{2}{|c|}{ Contrast algorithm $^{[17]}$} \\
\hline & & $\begin{array}{c}\text { Alarming } \\
\text { number }\end{array}$ & $\begin{array}{c}\text { Detection } \\
\text { rate[\%] }\end{array}$ & $\begin{array}{c}\text { Alarming } \\
\text { number }\end{array}$ & $\begin{array}{c}\text { Detection } \\
\text { rate[\%] }\end{array}$ \\
\hline Fighting & 74 & 72 & 97.30 & 68 & 94.44 \\
\hline Walking & 51 & 1 & 98.04 & 1 & 98.04 \\
\hline Talking & 30 & 1 & 96.67 & 2 & 93.33 \\
\hline Falling & 69 & 62 & 89.86 & 60 & 86.96 \\
\hline
\end{tabular}

\section{Summary}

In this paper the abnormal behavior detection method based on regional energy flow, is to describe the behavior and severity degree of confusion by unit energy flow, so that, to determine whether the act for the detection of abnormal behavior. Inferred from this essay, when the weighted energy histogram energy assignment increases suddenly and the continuation of a long time, it would be judged to have abnormal behavior; When a sudden increase in the weighted energy assignment but time is short, it cannot judge that there is no abnormal behavior. Through the simulation experiment, verify the reliability of experimental results further. But there are also limitations in test. For example, during the experiment, the main places selected in indoor that relatively environmental few changes. And the experiment will be more standardized.

Due to the ViBe algorithm not only reduces the process of background model, but also can deal the process that a sudden change in the background, so that in the foreground extraction, regardless 
of the position of the camera and its motion, the foreground extraction has certain robustness. At the same time through the optical flow energy to detect abnormal behavior, can adapt to different indoor and outdoor environment quickly, and do not rely on the sample library. Further will combination with other features for classification of abnormal behavior, used in public places.

\section{Acknowledgements}

National Natural Science Foundation of China(61272253). Science research project of Liaoning Provincial Department of Education L2015443. Ministry of housing project, 2015-K2-015

\section{References}

[1] POPPE R. Vision-based human motion analysis: an overview [J]. Computer Vision and Understanding, 2007, 104(2): 4-18.

[2] MITRA S, ACHARYA T. Gesture recognition: a survey [J]. IEEE Transaction on System, Man, and Cybernetics, Part C: Application and Reviews, 2007, 37(3): 311-324.

[3] MECOCCIA, PANNOZZOM. A completely autonomous system that learns anomalous movements in advanced video surveillance applications[C] // IEEE International Conference on Image Processing. London:IEEE, 2005: II-586-589.

[4] CALDERARA S, CUCCHIARA R, PRATI A. Detection of abnormal behaviors using a mixture of Von Mises distributions [C] // IEEE Conference on Advanced Video and Signal Based Surveillance. London: IEEE, 2007: 141-146.

[5] BOUTTEFROY P L M, BOUZERDOUM A, PHUNG SL, et al. Abnormal behavior detection using a multi-modal stochastic learning approach[C] // International Conference on Intelligent Sensors,Sensor Networks and Information Processing. Sydney: IEEE, 2008: 121-126.

[6] TEHRANI M A, KLEIHORST R, MEIJER P, et al.Abnormal motion detection in a real-time smart camera system[C] // 3rd ACM / IEEE International Conference on Distributed Smart Cameras. Como: IEEE, 2009: 1-7.

[7] XIANG Tao, GONG Shao-gang. Incremental and adaptive abnormal behavior detection[J]. Computer Vision and Image Understanding, 2008, 111 (1):59-73.

[8] FATHI A, MORI G. Action recognition by learning mid-level motion features[C] // IEEE Conference on Computer Vision and Pattern Recognition. Anchorage: IEEE, 2008: 1-8.

[9] DATTA A, SHAH M, LOBO N D V. Person-on-person violence detection in video data [C] // Proceedings of the 16th International Conference on Pattern Recognition. Quebec City: IEEE, 2002: 433-438.

[10]CHEN Yu-feng, LIANG Guo-yuan, LEE Ka-keung, et al. Abnormal behavior detection by multi-SVM-based Bayesian network[C] // International Conference on Information Acquisition. Seogwipo-si: IEEE, 2007: 298-303.

[11]M.Van Droogenbroeck and O.Barnich. ViBe: A Disruptive Method for Background Subtraction. In T. Bouwmans, F. Porikli, B. Hoferlin, and A. Vacavant, editors, Background Modeling and Foreground Detection for Video Surveillance, chapter 7. Chapman and Hall/CRC, June 2014.

[12] Barnich and M. Van Droogenbroeck. ViBe: A universal background subtraction algorithm for video sequences. In IEEE Transactions on Image Processing, 20(6): 1709-1724, June 2011. 
[13]M.Van Droogenbroeck and O. Paquot. Background Subtraction: Experiments and Improvements for ViBe. In Change Detection Workshop (CDW), Providence, Rhode Island, June 2012.

[14]Barnich and M.Van Droogenbroeck. ViBe: a powerful random technique to estimate the background in video sequences. In International Conference on Acoustics, Speech, and Signal Processing (ICASSP 2009), pages 945-948, April 2009.

[15]STAUFFER C, GRIMSON W E L. Adaptive background mixture models for real-time tracking [C] // Proceedings of Computer Vision and Pattern Recognition. Fort Collins: IEEE, 1999:246-252

[16]LUCAS B D, KANADE T. An iterative image registration technique with an application to stereo vision [C] // Proceedings of the 1981 DARPA IU Workshop. Washington DC: Morgan Kaufmann Publishers, 1981: 121-130.

[17] Jianhao Du, Li Xu. Abnormal behavior detection based on optical flow characteristics. Journal of Zhejiang University(Engineering Edition). 1008-973X. 2011.07.004. 